\title{
PHOTOBIOMODULATION UND COVID-19
}

\section{AUSWAHL VON STUDIENDESIGNS FÜR MÖGLICHE HEIM-BEHANDLUNG - KRITISCHE BETRACHTUNGEN}

\section{Photobiomodulation and COVID-19 \\ Selection of Study Designs for Possible Home Treatment - Critical Considerations}

\section{Zusammenfassung}

Die Zahl hochrangiger Publikationen zur Photobiomodulation steigt weltweit überproportional an und es ist daher auch leicht nachvollziehbar, dass die Therapieoption auch bei der Behandlung von COVID-19 als komplementäres Verfahren zur Pandemiebekämpfung methodisch erweitert und getestet werden soll. Um den gegenwärtigen Stand des Wissens zur Thematik zu erheben, wurde eine aktuelle Recherche zu klinischen laufenden Forschungsprojekten und den ersten vorliegenden Ergebnissen initiiert. Seitens der gewählten Methode wurde dabei die Variante einer möglichen selbst durchführbaren „Heim-Behandlung“ in den Fokus der Recherche gestellt. Drei aktuelle Studiendesigns sollen in diesem Übersichtsreport näher beschrieben und diskutiert werden. Die drei beschriebenen Studienvorhaben entsprechen zwar nicht alle den seitens einer ,good scientific practice“ notwendigen Anforderungen, sollen aber dennoch aufgrund der Aktualität hier kurz vorgestellt werden. Wenngleich die Designs durchaus wissenschaftlich beurteilbare Ergebnisse erwarten lassen, kann derzeit (noch) kein wissenschaftlicher Schluss gezogen werden, dass sich die drei Verfahren zur Behandlung von COVID-19 als geeignet erweisen. Gründe dafür sind, dass bei zwei der drei Studien noch keine Ergebnisse vorliegen und eine Pilotstudie grobe formale wissenschaftliche Mängel aufweist, die es gilt, in Folgeuntersuchungen zu vermeiden.

\section{Schlüsselwörter}

Photobiomodulation, COVID-19, SARS-CoV-2, Laseruhr, Riboflavin, Grundlagenforschung, evidenzbasierte Komplementärmedizin, integrative Medizin

\section{Summary}

The number of high-ranking publications on photobiomodulation is increasing disproportionately worldwide and it is therefore easy to understand that the therapy option should also be methodically expanded and tested in the treatment of COVID-19 as a complementary method for combating pandemics. In order to ascertain the current state of knowledge on the subject, a current research on ongoing clinical research studies and the first available results was initiated. In terms of the chosen method, the research focused on the variant of a possible self-executable home treatment. Three current study designs are to be described and discussed in more detail in this overview report. The three study projects do not all meet the requirements of good scientific practice, but should be briefly presented here because they are up to date. Although the designs can be expected to provide scientifically assessable results, no scientific conclusion can currently (yet) be drawn that the three methods for the treatment of COVID-19 prove to be suitable. The reasons for this are that no results are yet available in two of the three studies and one pilot study has major formal scientific deficiencies that should be avoided in follow-up studies.

\section{Keywords}

photobiomodulation, COVID-19, SARS-CoV-2, laser watch, riboflavin, basic research, evidence-based complementary medicine, integrative medicine 


\section{Einleitung}

Weltweit werden mit Beginn des Jahres 2021 die lang ersehnten ersten Impfungen gegen COVID-19 in größeren Rahmen durchgeführt und viele Menschen haben Hoffnung, dass es in diesem Jahr wieder zu einer sogenannten Normalisierung der Lebensweise kommen wird. Keineswegs darf jedoch aus Sicht des Autors der Einschätzungsfehler gemacht werden, dass mit der großartigen und raschen Entwicklung der ersten Impfstoffe die Pandemie plötzlich zu Ende ist und alle Probleme in diesem Zusammenhang zur Gänze gelöst sind. Das menschliche Immunsystem in Nase und Rachen beinhaltet auch andere Aspekte als im Rest des Körpers. Daher scheint es wichtig im Hinblick auf weitere Forschungsaktivitäten, gegebenenfalls Stoffe und/oder Behandlungsmethoden zu entwickeln, die eventuell über die Nase verabreicht werden können. Es könnte durch Mutationen auchVirusvarianten von SARS-CoV-2 geben, für die die entwickelten Impfstoffe nicht ausreichend Schutz bieten, wenngleich dies im Moment (etwa bei B.1.1.7) noch eher unwahrscheinlich erscheint. Impfstoffe sind aus Sicht des Autors absolut notwendig und essenziell, aber, wie aus zahlreichen Diskussionen hervorgeht, nicht die einzige Lösung zur umfassenden Pandemiebekämpfung. Umfangreiche Tests sowie Medikamente und Behandlungsmethoden für COVID19-Patient ${ }^{\star}$ innen werden nach wie vor benötigt, um die schwerste gesundheitliche Krise der letzten Jahrzehnte nachhaltig unter Kontrolle zu bringen.

Im Rahmen dieses Beitrags sollen drei ausgewählte Beispiele von Studiendesigns für mögliche Behandlungsmethoden aus dem Bereich der Photobiomodulation (PBM) wie beispielsweise Nasen-, Thorax-, Rachen- und Blutstimulation aufgelistet werden, die eventuell nach erfolgreichen ersten klinischen Testphasen als zusätzliche Therapieoption bei COVID-19 in Betracht gezogen werden könnten [1-5].

\section{Methode}

Wissenschaftliche klinische Studiendesigns und klinische Studien aus den Datenbanken ClinicalTrials.gov der U.S. National Library of Medicine, PubMed und Google Scholar dienten als Forschungsdokumente. Insgesamt wurden drei relevante Quellen [6-8] identifiziert, von denen sich zwei noch in der ersten Testphase befinden.

\section{Ergebnisse}

\section{Studiendesign - Universität Kairo [6]}

Am 4. Juni 2020 reichte die Universität Kairo eine klinische Studie mit dem Titel „Photodynamische Therapie

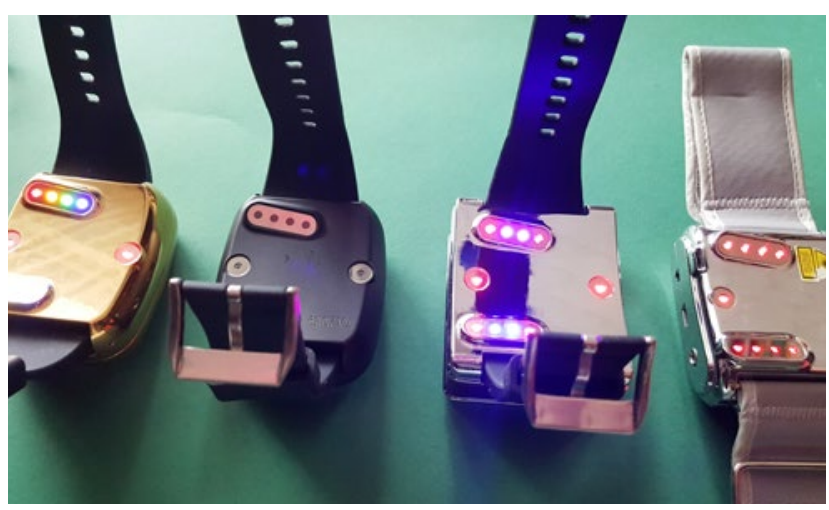

Abb. 1: Laserblutbestrahlung und Laserakupunktur über eine sogenannte Laseruhr. Dargestellt sind exemplarisch 4 unterschiedliche Gerätegenerationen der Uhr.

zur Behandlung von COVID-19“ mit der Identifikationsnummer NCT04416113 ein [6]. Die Forscher ${ }^{\star}$ innen berichten in der Antragsstellung, dass die grundlegenden Mechanismen der nicht invasiven Low-Level-Lasertherapie, der PBM und der photodynamischen Therapie (PDT) darin bestehen, die mitochondriale Atmungskette zu stimulieren, wobei eine vorübergehende Freisetzung nicht zytotoxischer Spiegel reaktiver Sauerstoffspezies zu einer positiven Modulation der Antwort des Immunsystems führt. Die aus der Sicht der Antragsteller ${ }^{\star}$ innen wichtigste Strategie für das COVID-19-Management besteht in der Sauerstoffanreicherung und schnelleren Rehabilitation des geschädigten Gewebes, den antiviralen Wirkungen und schließlich in der Reduzierung oder Kontrolle eines Zytokinsturms durch Reduzierung der Entzündungserreger. Ziel dieser klinischen Studie ist es daher zu prüfen, ob die Photobiomodulationstherapie (PBMT) bzw. die PDT als zusätzliche Behandlungsmethode ohne Nebenwirkungen und Arzneimittelwechselwirkungen für COVID-19-Patient`innen eingesetzt werden können.

Die randomisierte kontrollierte Studie soll an insgesamt 60 Patient $\star_{\text {innen }}$ mit COVID-19-Erkrankung durchgeführt werden. Die Einteilung der Personen erfolgt dabei in drei gleiche Gruppen:

- Gruppe 1 wird eine Low-Level-Laser-Therapie (Light emitting diodes, LED; Diodenlaser $980 \mathrm{~nm}$ ) mittels einer sogennanten „Laser Watch“ [5] (Laseruhr; Abb. 1) für die Dauer von 30 Minuten, mit jeweils $20 \mathrm{~J}$ für 3-5 Tage erhalten. Zusätzlich wird dieser Gruppe ebenfalls über die Laseruhr eine Laserakupunktur verabreicht.

- Gruppe 2 wird mit einer PDT behandelt, indem Methylenblau (0,1-0,2 $\mathrm{ml}$ 1\%ige Lösung pro Kilogramm Körpergewicht) als Photosensitizer über einen Zeitraum von mehreren Minuten sehr langsam intravenös injiziert 
Abb. 2a u. b: Vielight

Laserstimulationsequipment zur

Heim-Anwendung (mit freundlicher Genehmigung von Vielight Inc, Toronto, Kanada, Gennady Lemud MBA CIM, Communications Director Vielight Inc.)

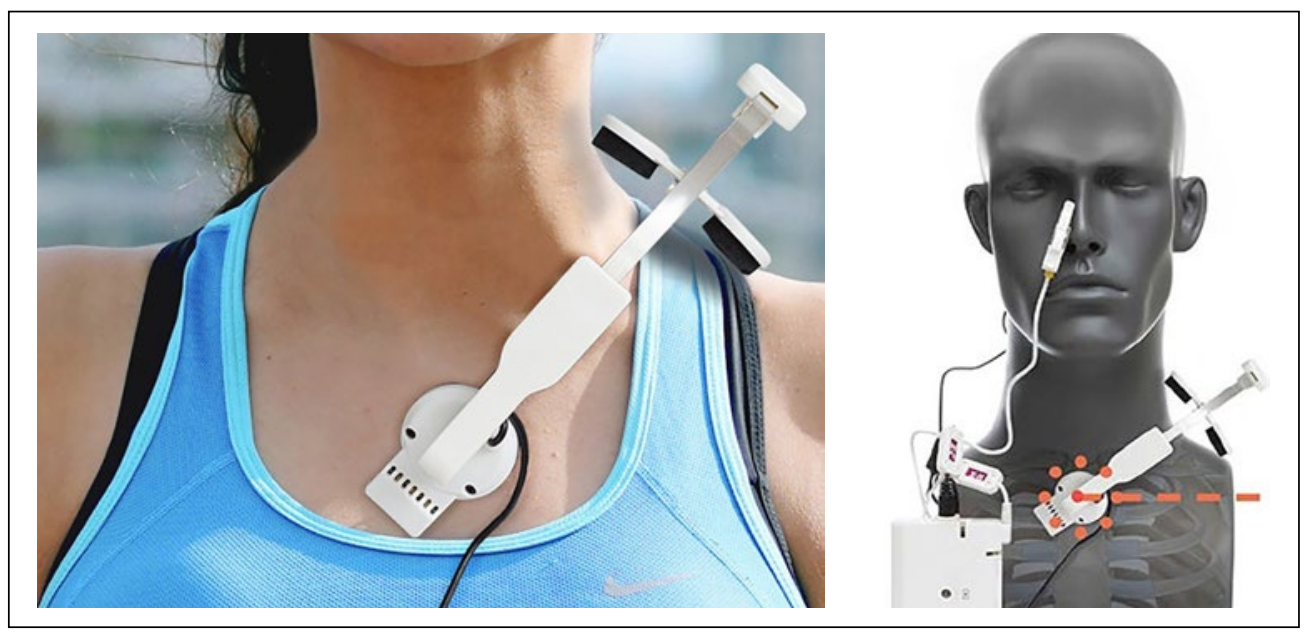

und anschließend mit einer Laseruhr (Diodenlaser 670 $\mathrm{nm})$ bestrahlt wird.

- Gruppe 3 dient als Kontrolle ohne PDT bzw. Laserbestrahlung.

Die Bewertungsmethoden umfassen Laboruntersuchungen und Thorax-CT.

Bei der Studie der Universität Kairo handelt es sich um eine randomisierte, interventionelle klinische Studie mit paralleler Zuordnung. Der Beginn der Untersuchungen war August 2020 und das voraussichtliche Abschlussdatum der Studie wird mit Februar 2021angegeben. Stimuliert werden die A. radialis und die A. ulnaris sowie die Akupunkturpunkte Neiguan (KS 6) und Daling (KS 7).

Die Dosis soll laut Angaben insgesamt $100-200 \mathrm{~J} / \mathrm{cm}^{2}$ betragen. Die Behandlungen werden zweimal pro Woche durchgeführt. In die Studie werden sowohl Kinder, Erwachsene und auch ältere Erwachsene mit einbezogen. Als Einschlusskriterien werden Krankenhauspatient $\star^{\star}$ innen mit positivem COVID-19-Test, welche unter Fieber, Husten, Atemnot, Schüttelfrost, Muskelschmerzen, Geschmacksoder Geruchsverlust, Erbrechen oder Durchfall und/oder Halsschmerzen leiden, angegeben. Die Ausschlusskriterien

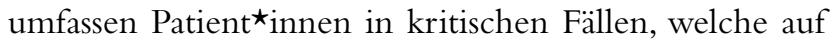
der Intensivstation aufgenommen wurden, bakterielle Lungenentzündung und Atemnot Grad III und IV sowie Patient ${ }^{\star}$ innen mit einem mechanischen Beatmungsgerät. Ergebnisse wurden zum gegenwärtigen Zeitpunkt (13. Jänner 2021) noch keine publiziert.

\section{Studiendesign - Health Canada [7]}

Nur einen Tag nachdem das Design von der Universität Kairo offiziell eingereicht wurde, wurde am 5. Juni 2020 eine weitere klinische Studie mit dem Titel „Vielight RX
Plus zur Behandlung von COVID-19-Atmungssymptomen (COVIDLight)“ mit der Identifikationsnummer NCT04418505 in den USA eingereicht [7]. Sponsor dieser Studie ist die Firma Vielight Inc. aus Toronto in Kanada.

In dieser Studie soll unter anderem ebenfalls die Hypothese getestet werden, dass die Genesung von Patient ${ }^{\star}$ innen, die positiv auf eine COVID-19-Infektion getestet wurden, durch eine spezielle Lichttherapie beschleunigt werden kann. Bei der Studienintervention wird eine Kombination aus intranasal verabreichtem LEDRotlicht und LED-basiertem Nahinfrarotlicht verabreicht. $\mathrm{Zu}$ diesem Zweck wurde ein kompaktes und tragbares Gerät entwickelt, welches auch für eine sogenannte Heim-Anwendung geeignet sein soll. Das System basiert auch auf Infrarot- und Rotlichtstimulation über LEDs, die direkt am Manubrium des Sternums, das die Thymusdrüse bedeckt, bzw. in einem Nasenloch angebracht sind (Abb. 2).

Die aufgestellte Hypothese wird in einer randomisierten kontrollierten klinischen Studie getestet werden. Die COVID-19-Patient ${ }^{\star}$ innen aus den USA sollen zwischen 18 und 65 Jahre alt sein. Die Studie wird im Sinne einer Telestudie in der Quarantäne durchgeführt.Videokonsultationen werden für die Kommunikation mit den Studienteilnehmer ${ }^{\star}$ innen bereitgestellt. Im Rahmen der Studie werden 280 Teilnehmer ${ }^{\star}$ innen untersucht werden. 140 Personen werden nach dem aktiven Laser-Protokoll und die anderen 140 Teilnehmer ${ }^{\star}$ innen nach dem Standard-Versorgungsschema randomisiert. Die Rekrutierung von Teilnehmer ${ }^{\star}$ innen ist derzeit noch offen und noch nicht abgeschlossen.

Alle Daten werden elektronisch aus Online-Fragebögen erfasst. Die Unterstützung und Überwachung werden 
durch medizinisch qualifiziertes Personal erfolgen. Es wird kein persönlicher Kontakt stattfinden. Der mögliche Erfolg dieser Studie wird an der Zeit bis zur Genesung über einen Studienzeitraum von 30 Tagen gemessen werden.

Als Einschlusskriterien werden eine bestätigte COVID19-Infektion, das Auftreten mittelschwerer bis schwerer Atemwegsbeschwerden und ein Alter von 18-65 Jahren angegeben. Ausschlusskriterien sind die Notwendigkeit eines Krankenhausaufenthalts zum Zeitpunkt der Diagnose und/oder Bedarf an zusätzlicher Sauerstoff- oder Überdruckunterstützung für eine Zeitdauer über 24 Stunden, das Verstreichen von mehr als 10 Tagen seit Auftreten der ersten Symptome, die Diagnose einer chronisch-obstruktiven Lungenerkrankung (COPD), Schwangerschaft, ein positiver Test auf Hepatitis-C-Virus (HCV), HepatitisB-Virus (HBV) oder Human Immunodeficiency Virus (HIV) sowie die Unfähigkeit, Studienfragebögen auf Englisch elektronisch auszufüllen. Das voraussichtliche Abschlussdatum der Studie wird mit 1. März 2021 angegeben, Zwischenergebnisse wurden bislang noch nicht einer breiten Öffentlichkeit zugänglich gemacht.

\section{Studiendesign und erste Ergebnisse - Ghandhi Hospital Teheran [8]}

Gleich zu Beginn der Erklärungen dieser bereits durchgeführten und veröffentlichten klinischen Pilotstudie soll darauf hingewiesen werden, dass sich in der Arbeit keine Hinweise auf eine Registrierung der klinischen Studie finden. Die Studie konnte vom Autor des vorliegenden Berichts in keinem Studienregister als gemeldet gefunden werden. Dennoch scheint die Pilotstudie erwähnenswert, da das zugrunde liegende Design eine klare Erweiterung der beiden erstgenannten registrierten Studien beinhaltet [8].

Die Studie bestand aus zwei Gruppen (Verum und Kontrolle) mit jeweils 20 Patient $\star^{\star}$ innen, wobei die Versuchsgruppe eine PDT inklusive täglichen Tests erhielt. Alle Patient $\star^{\star}$ innen in beiden Gruppen waren positiv auf COVID-19 getestet und befanden sich in einem frühen Infektionsstadium mit leichten Symptomen wie Fieber, trockenem Husten, Kopfschmerzen, schweres Atmen und Müdigkeit. PCR-Tests wurden am Tag 1, 2, 3, 4, 5 und 7 in der Patient ${ }^{\star}$ innengruppe und am Tag 1,3,5 und 7 in der Kontrollgruppe durchgeführt.

Das Studienequipment (Abb. 3) wurde von Weber Medical GmbH (Deutschland) entwickelt und bereitgestellt und bestand aus je 60 Kapseln Riboflavin-5-phosphat 100 mg, einem Lichtbehandlungsgerät - „Laseruhr Spectra“ mit 4 roten $(658 \mathrm{~nm}), 2$ blauen (447 nm), 2 grünen (532 $\mathrm{nm})$ und 2 gelben $(589 \mathrm{~nm})$ Laserdioden für eine systemi- sche Behandlung von Blut über die Handgelenkarterien (A. radialis und A. ulnaris) - sowie Laserakupunktur, einer Sprühflasche mit Mund- und Nasenapplikator, einem Nasenbehandlungsapplikator mit einer blauen LED (447 nm) und einer UVA LED (375 nm), einem Mund- bzw. Rachenbehandlungsapplikator mit 14 blauen LEDs (447 nm) und 14 UVA-LEDs (375 nm).

Der Rachenapplikator hat ein gekrümmtes Ende und ermöglicht es somit, tief in den Hals zu bestrahlen. Die Laseruhr zur systemischen Behandlung und Stimulation des zirkulierenden Riboflavin wird am Handgelenk befestigt, damit das Licht in die Arterien eindringen kann.

Die Autor^innen aus Deutschland und dem Iran haben sich für einen nicht invasiven Ansatz entschieden, da sie auch wie im vorgenannten Design das System als „HomeUse-System“ einsetzen möchten. Am wichtigsten scheint in diesem Studiendesign wohl die systemische Stimulation des zirkulierenden Riboflavins durch die blauen Dioden $(447 \mathrm{~nm})$. Die photodynamische Therapie über das Blut via Handgelenkarterien könnte - so wird zumindest in der Arbeit spekuliert - auch eine vorbeugende Wirkung haben [8].

Bei Fortschreiten der Erkrankung sieht das Behandlungsprotokoll die Einnahme einer Kapsel Riboflavin5-phosphat (100 mg) zu einer Mahlzeit vor. Danach soll eine zweite Kapsel (100 mg) geöffnet werden und in einem Glas mit $200 \mathrm{ml}$ Wasser (zur lokalen Anwendung in Nase, Mund und Rachen) aufgelöst werden. Nach einer Stunde nach der Einnahme soll das Lichtbehandlungsgerät (,,Spectra Watch") zum Einsatz kommen. Es wird am Handgelenk appliziert und liefert für die Dauer von 60 Minuten eine optische Reizdarbietung. Zusätzlich soll über eine Sprühflasche die Riboflavinlösung dreimal in beide Nasenlöcher gesprüht werden. Mit der restlichen Lösung soll der Mund gespült sowie gegurgelt werden. Auch empfehlen die Autor ${ }^{\star}$ innen das Trinken der Lösung. Anschließend wird

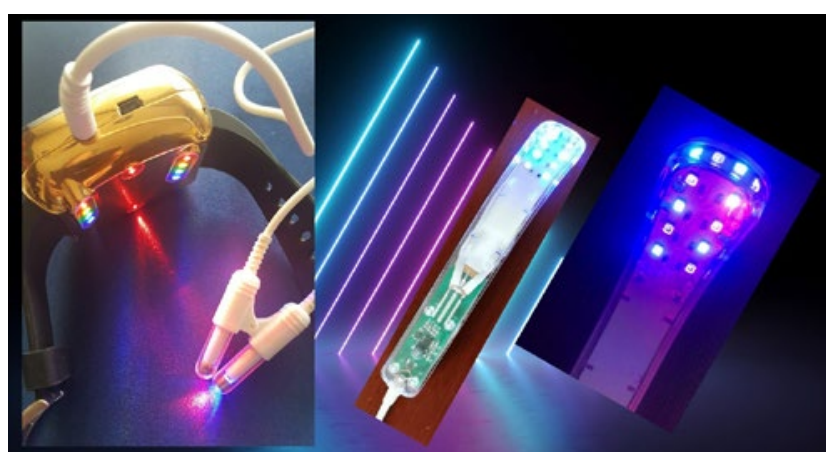

Abb. 3: Studienequipment für die Pilotstudie in Teheran von Weber Medical GmbH, Lauenförde, Deutschland und Suyzeko Ltd., Shenzhen, China 
der Nasen- und Mundapplikator befestigt bzw. angewendet. Jedes Nasenloch soll 10 Minuten lang mit Blau- und UVA-Licht und der Mund und Rachenraum 20 Minuten lang stimuliert werden.

Der Schwerpunkt des vorgestellten Behandlungsprotokolls liegt auf der Behandlung der infizierten Bereiche wie Nase, Mund und Rachen. Zusätzlich soll ein systemischer Effekt durch Applikation der Lichtstimulation an den Handgelenkarterien über die Laseruhr erreicht werden. In die Untersuchungen wurden Männer und Frauen inklusive Jugendlicher zwischen 10 und 90 Jahren eingeschlossen. Ausgeschlossen waren schwangere Frauen. Die Verumgruppe bestand aus 20 Patient $\star^{\star}$ innen. Alle hatten positive PCR-Tests und Covid-19-bezogene Symptome wie Fieber, Halsschmerzen, Husten etc. Kein ${ }^{\star}$ e Patient ${ }^{\star}$ in musste mit Sauerstoff versorgt werden. Eingeschlossen wurden letztendlich 13 Frauen und 7 Männer im Alter zwischen 18 und 80 Jahren. 20 weitere Patient ${ }^{\star}$ innen von derselben Klinik in Teheran dienten als Kontrollgruppe für diese Studie (12 Frauen und 8 Männer, im Alter zwischen 22 und 77 Jahren). Sie wurden nach konventionellen Behandlungsmodalitäten gemäß den im Iran empfohlenen Standards für das Covid-19-Management behandelt. Auf die PDT wurde bei dieser Gruppe verzichtet.

Alle 20 Patient ${ }^{\star}$ innen in derVerumgruppe zeigten eine signifikante Verbesserung der klinischen Symptome und in der Beurteilung derViruslast innerhalb der ersten 5 Tage nach der PDT-Behandlung. 14 von 20 Patienten hatten nach 5 Tagen Behandlung mit PDT einen negativen PCRTest, während die restlichen 6 Patient ${ }^{\star}$ innen ebenfalls eine signifikant verringerte Viruslast aufwiesen. Bei den 20 Patient $\star^{\star}$ innen der Kontrollgruppe mit konventioneller Pflege konnte innerhalb von 5 Tagen keine signifikante Verbesserung festgestellt werden [8].

Die Autor ${ }^{\star}$ innen ziehen folgende Schlussfolgerung und berichten, dass in der Pilotstudie der Nachweis erbracht wurde, dass die PDT bei COVID-19 Erfolg zeigen kann. Dies kann sowohl hinsichtlich derVerringerung derViruslast als auch bei derVerbesserung der klinischen Symptome und der Verhinderung von Krankenhausaufenthalten und Intensivbehandlungen zielführend sein. Sie stellen weiters fest, dass die Behandlung einfach und kostengünstig durchzuführen ist und zu Hause erfolgen kann [8].

In der Folge sollen nun umfangreiche Studien durchgeführt werden. Inwieweit diese klinischen Studien bereits (Stand 13. Jänner 2021) einer Registrierung zugänglich gemacht oder beantragt wurden, ist dem Autor dieses Reports nicht bekannt. Nach dessen Ansicht kann selbstverständlich nur durch eine sorgsame und gesetzeskonforme
Vorgangsweise und durch eine international anerkannte (peer review) Publikation der Einsatz des neuenVerfahrens beiVorliegen entsprechender Ergebnisse für eine mögliche breitere Anwendung herangezogen werden.

\section{Diskussion und Zukunftsaspekte}

Dr. Michael Hamblin, außerordentlicher Professor Emeritus an der Harvard Medical School und am Massachusetts General Hospital stellt 2020 als Top-Experte im Bereich der PBM zur Thematik folgendes fest: „Die Gesamtheit der veröffentlichten Forschungsergebnisse über PBM und die damit verbundene Ätiologie der Coronavirus-Infektion legen nahe, dass PBM die Replikation von Coronaviren wirksam hemmen könnte. Es könnten die Aktivitäten des Immunsystems angekurbelt und gleichzeitig das Entzündungsrisiko beherrscht werden“" [7].

Die PBM wird von mehreren Forscher^innen als mögliches Verfahren zur Symptomlinderung und Behandlung bei COVID-19-Erkrankungen empfohlen bzw. diskutiert. Beispielsweise berichten Liebert u. Mitarb. 2020 [1], dass sich PBM über viele Jahre als sichere, wirksame, nicht invasive und leicht einsetzbare Zusatzbehandlungsoption für entzündliche Erkrankungen, Schmerzen oder Wundheilung erwiesen hat. PBM kann möglicherweise auch für diejenigen COVID-19-Patient ${ }^{\star}$ innen von Vorteil sein, die mit demVirus infiziert sind und keine schweren Symptome aufweisen, aber auch für die, die sich nur langsam von den Auswirkungen des Virus erholen [1].

Eine Übersicht über die derzeit existierenden anerkannten wissenschaftlichen Publikationen mit Relevanz zum Titel dieser Arbeit findet sich in der Tab. 1.

Die einzige bislang veröffentlichte systematische Übersichtsarbeit zur Thematik PBM und COVID-19 wurde im Jänner 2021 von Nejatifard u. Mitarb. [9] veröffentlicht und beinhaltet insgesamt 17 relevante Artikel. Wichtig scheint hier anzumerken, dass es sich dabei ausschließlich um Arbeiten an Tiermodellen handelt. Die Ergebnisse zeigten laut Angaben der Autor ${ }^{\star}$ innen, dass das PBM hilfreich sein könnte, die COVID-19-Erkrankung zu reduzieren und die Regeneration des geschädigten Gewebes zu fördern. PBM war im Stande, die Sauerstoffversorgung indirekt zu erhöhen, um die betroffenen Organe zu regenerieren. Die Autor ${ }^{\star}$ innen empfehlen, Infrarotlaser oder LEDs zu verwenden [9].

„Zieht man die Pathophysiologie von COVID-19 und mögliche positive Auswirkungen einer PBMT auf die Funktion des Immunsystems in Betracht, könnte diese Behandlungsmethode bei schweren COVID-19-Fällen wirksam sein“. So berichten Soheilifar u. Mitarb. [10] in 
Tab. 1: Wissenschaftliche Arbeiten zur Thematik Photobiomodulation und COVID-19 (Stand 13.1.2021)

\begin{tabular}{|c|c|c|c|c|}
\hline $\begin{array}{l}\text { Wissenschaftliche Arbeiten } \\
\text { (Photobiomodulation und } \\
\text { COVID-19) }\end{array}$ & $\begin{array}{c}\text { Anzahl } \\
\text { (PubMed) }\end{array}$ & $\begin{array}{l}\text { Monat/Jahr der } \\
\text { Veröffentlichung }\end{array}$ & $\begin{array}{l}\text { Autor*innen und } \\
\text { Referenz }\end{array}$ & Herkunft der Autor*innen \\
\hline Systematische Übersichtsarbeit & 1 & Jan/2021 & Nejatifard M. et al. [9] & Iran, Südafrika (USA)* \\
\hline Übersichtsarbeit & 4 & $\begin{array}{l}\text { Nov/2020 } \\
\text { Dez/2020 } \\
\text { Sep/2020 } \\
\text { Nov/2020 }\end{array}$ & $\begin{array}{l}\text { Soheilifar S. et al. [10] } \\
\text { Surazakov A. et al. [11] } \\
\text { Hanna R. et al. [12] } \\
\text { Sabino C.P. et al. [13] }\end{array}$ & $\begin{array}{c}\text { Iran, Kanada } \\
\text { Zypern, Russland } \\
\text { Italien, England, Indien, } \\
\text { Rumänien } \\
\text { Brasilien, USA, Südafrika, } \\
\text { Spanien, England }\end{array}$ \\
\hline $\begin{array}{l}\text { Randomisierte kontrollierte } \\
\text { Studie }\end{array}$ & 0 & - & - & - \\
\hline Metaanalyse & 0 & - & - & - \\
\hline Klinische Studie & 0 & - & - & - \\
\hline Bücher & 0 & - & - & - \\
\hline $\begin{array}{l}\text { Anderer Artikel (Editorial, } \\
\text { Fallbericht, etc.) }\end{array}$ & 13 & Apr-Dez/2020 & siehe PubMed & siehe PubMed \\
\hline
\end{tabular}

*) Für den in erster Linie in den USA in Boston tätigen Prof. Michael Hamblin wurde in dieser Arbeit eine Korrespondenzadresse der Universität Johannesburg (Südafrika) angegeben.

einer Übersichtsarbeit. Sie stellen weiters fest, dass die Effekte der PBM bei COVID-19-Patient ${ }^{\star}$ innen wirksam sein können und diese nur geringe Nebenwirkungen hat. Die Hypothese der Autor^innen basiert jedoch hauptsächlich auf theoretischen Daten. Daher schlagen sie vor, dass Forscher^innen das mögliche Potenzial dieser Behandlungsmethode erst ausloten sollen, um damit eventuell das Leben schwer betroffener Patient ${ }^{\star}$ innen retten zu können.

Auch Hanna u. Mitarb. [12] schreiben, dass die Phototherapie eine vielversprechende Behandlungsmethode für COVID-19 sein könnte, und durch groß angelegte randomisierte, doppelblinde, placebokontrollierte klinische Studien validiert werden sollte, um valide Ergebnisse zu erhalten. Ähnlich auch die Kommentare von Sabino u. Mitarb. [13]. Sie berichten, dass lichtbasierte Technologien ein nachweislich breites Wirkungsspektrum aufweisen und zeigen lichtbasierte Strategien zur Bekämpfung der COVID-19-Pandemie auf.

Neueste Studien berichten darüber, dass eine PBMT bei COVID-19 von Nutzen sein kann, indem das Blut entweder direkt oder transdermal und/oder gezielt nur die Lunge behandelt wird [1,14-17]. Die PBM-Therapie wird nicht nur, wie im vorliegenden Report aufgezeigt, in den USA, Ägypten und dem Iran, sondern auch in Russland als COVID-19-Therapie getestet [1]. Darüber hinaus wurde erst neulich ein Bericht über die Wirksamkeit der PBM-Therapie bei der Behandlung eines Patienten mit schwerer COVID-19-Erkrankung aus Afrika veröffentlicht [17].

Trotz erster positiver Aspekte dieser Behandlungsart muss jedoch abschließend festgestellt werden, dass es bislang keine einzige wissenschaftlich anerkannte größere klinische Studie gibt (s. Tab. 1), die gesicherte Ergebnisse in Bezug auf eine mögliche Wirksamkeit der PBM bei COVID-19 liefert.

\section{Danksagung}

Der Artikel wurde nach Vorschlag von Prof. Frank Bahr aufgrund einer Einladung des Redaktionsteams von Akupunktur \& Aurikulomedizin erstellt. Gerne ist der Autor dem Wunsch nachgekommen, einige neue Möglichkeiten der PBM aus wissenschaftlicher Sicht zu dokumentieren. Er möchte sich bei folgenden Personen für die Überlassung von Informationsmaterial zur Thematik bedanken: Gennady Lemud MBA CIM, Communications Director,Vielight Inc. Toronto, Kanada und Dr. med. Dipl. chem. Hans Michael Weber, Laser Therapy and Research Center, Lauenförde, Deutschland.

Interessenkonflikte: Der Autor erklärt keinen Interessenkonflikt. 


\section{Literatur}

[1] Liebert A, Bicknell B, Markman W et al.: A potential role for photobiomodulation therapy in disease treatment and prevention in the era of COVID-19.Aging Dis 2020;11:1352-1362.

[2] Litscher G: Can laser medicine and laser acupuncture be used for COVID-19? Selected areas of the current scientific literature. OBM Integrative and Complementary Medicine 2020; $5: 6$.

[3] Liang FX, Litscher G: COVID-19 (Coronavirus Disease-19): Traditional Chinese Medicine including acupuncture for alleviation - a report from Wuhan, Hubei Province in China. OBM Integrative and Complementary Medicine 2020; 5: 4.

[4] Litscher G: Effectiveness of integrative medicine in COVID-19? Med Acupunct 2020; 32: 131-132.

[5] Litscher G, Litscher D: A laser watch for simultaneous laser blood irradiation and laser acupuncture at the wrist. Integr Med Int 2016; 3: 75-81.

[6] Photodynamic therapy for the treatment of COVID-19. https://clinicaltrials.gov/ct2/show/NCT04416113. Stand: 14.1.2021.

[7] Vielight RX Plus for the treatment of COVID-19 respiratory symptoms (COVIDLight). https://www.clinicaltrials.gov/ ct 2 / s how / NCT04418505 ? cond = covid $19+$ vielight $\&$ draw $=2 \&$ rank=1. Stand: 14.1.2021.

[8] Weber HM, MehranYZ, Orthaber A et al.: Successful reduction of SARS-CoV-2 viral load by photodynamic therapy (PDT) verified by QPCR - a novel approach in treating patients in early infection stages. Med Clin Res 2020; 5: 311325 .

[9] Nejatifard M,Asefi S, Jamali R et al.: Probable positive effects of the photobiomodulation as an adjunctive treatment in COVID-19: A systematic review. Cytokine 2021; 137: 155312.
[10] Soheilifar S, Fathi H, Naghdi N: Photobiomodulation therapy as a high potential treatment modality for COVID-19. Lasers Med Sci 2020; 25: 1-4.

[11] Surazakov A, Klassen A, Gizinger O: The bioenergetics of COVID-19 immunopathology and the therapeutic potential of biophysical radiances. J Photochem Photobiol B 2020;213: 112083.

[12] Hanna R, Dalvi S, Sălăgean T et al.: Phototherapy as a rational antioxidant treatment modality in COVID-19 management; new concept and strategic approach: critical review. Antioxidants (Basel) 2020; 9: 875.

[13] Sabino CP, Ball AR, Baptista MS et al.: Light-based technologies for management of COVID-19 pandemic crisis. J Photochem Photobiol B 2020; 212: 111999.

[14] Fernandes AB, de Lima CJ,Villaverde AGJB et al.: Photobiomodulation: shining light on COVID-19. Photobiomodul Photomed Laser Surg. 2020; 38: 395-397.

[15] Domínguez A, Velásquez SA, David MA: Can transdermal photobiomodulation help us at the time of COVID-19? Photobiomodul Photomed Laser Surg 2020; 38: 258-259.

[16] Fekrazad R: Photobiomodulation and antiviral photodynamic therapy as a possible novel approach in COVID-19 management. Photobiomodul Photomed Laser Surg 2020; 38: 255257.

[17] Sigman SA, Soheila M, Monica M et al.:A 57-year-old African American man with severe COVID-19 pneumonia who responded to supportive photobiomodulation therapy (PBMT): first use of PBMT in COVID-19. Am J Case Rep 2020; 21: e926779.

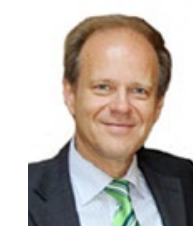

Univ.-Prof. Prof. mult. DI DDr. Gerhard Litscher

Leiter der Forschungseinheit für Biomedizinische Technik in Anästhesie und Intensivmedizin, der Forschungseinheit für Komplementäre und Integrative Lasermedizin

Chairman des TCM Forschungszentrums Graz, Medizinische Universität Graz

Auenbruggerplatz 39,EG19, 8036 Graz, Österreich, E-Mail:gerhard.litscher@medunigraz.at 